\title{
Reducing feather pecking and cloacal cannibalism by providing layer breeders with nest boxes in colony cages for natural mating
}

\author{
Haipeng Shi ${ }^{1,2,3}$, Weichao Zheng ${ }^{1,2,3}$, Jiang $\mathrm{Tu}^{1,2,3}$, Baoming $\mathrm{Li}^{1,2,3 *}$ \\ (1. College of Water Resources and Civil Engineering, China Agricultural University, Beijing 100083, China; \\ 2. Key Laboratory of Agricultural Engineering in Structure and Environment, Ministry of Agriculture and Rural Affairs, Beijing 100083, \\ China; 3. Beijing Engineering Research Center for Livestock and Poultry Healthy Environment, Beijing 100083, China)
}

\begin{abstract}
Colony cages for natural mating have been applied on many layer breeder farms. However, feather pecking and cloacal cannibalism are considered as prominent problems for these systems. This study aimed to investigate the effects on feather pecking and vent pecking behaviors by providing layer breeders with nest boxes $(0.90 \mathrm{~m} L \times 0.40 \mathrm{~m} \mathrm{~W} \times 0.60 \mathrm{~m} \mathrm{H})$ in colony cages for natural mating $(4.80 \mathrm{~m} L \times 1.20 \mathrm{~m} \mathrm{~W} \times 0.71 \mathrm{~m} \mathrm{H})$. Video recording and artificial observation were adopted. The results showed that the usage of nest boxes gradually increased from the beginning of nest boxes providing at the age of 37 weeks to the end of the experiment at the age of 55 weeks. The utilization rates of nest boxes were $36.75 \%$, $44.75 \%$ and $50.38 \%$ at the ages of 41 weeks, 47 weeks and 53 weeks, respectively. The hens in the cages without nest boxes showed significantly more frequent gentle feather pecks (both given and received) than hens in the cages with nest boxes at the ages of 41 weeks, 47 weeks and 53 weeks. A decreasing trend of gentle feather pecking frequency (both given and received) was found with the increasing age for both the two groups. No significant difference was found in the number of given or received severe feather pecks at the age of 41 weeks. The hens in the cages without nest boxes showed a significantly higher frequency of given and received severe feather pecks than hens in the cages with nest boxes at the ages of 47 weeks and 53 weeks $(p<0.05)$. Both cannibalistic injury occurrence and mortality rate in the cages with nest boxes were significantly lower than those in the cages without nest boxes. In conclusion, providing nest boxes in colony cages for natural mating could decrease abnormal pecking behaviors and improve animal welfare conditions of layer breeders.
\end{abstract}

Keywords: feather pecking, vent pecking, nest box, colony cage, cloacal cannibalism, layer breeder, animal welfare DOI: $10.25165 /$ j.ijabe. 20181106.3323

Citation: Shi H P, Zheng W C, Tu J, Li B M. Reducing feather pecking and cloacal cannibalism by providing layer breeders with nest boxes in colony cages for natural mating. Int J Agric \& Biol Eng, 2018; 11(6): 27-32.

\section{Introduction}

Due to increasing labor cost, an alternative housing system with unfurnished colony cages for natural mating of parent-stock (PS) layer breeders has been widely applied in China, instead of the conventional cages for artificial insemination. However, high mortality caused by vent pecking (VP) and poor plumage condition resulting from feather pecking (FP) were found in the system, which subsequently reduced the fertility rate of layer breeders and caused severe health, welfare, and economic problems. Reducing the FP and VP behaviors in the alternative system has become a crucial issue in the industry.

Feather pecking is characterized as pecking at and pulling out of feathers of conspecifics ${ }^{[1]}$. It varies from gentle FP to severe FP or feather pulling, which causes feather damage and feather $\operatorname{loss}^{[2]}$. Vent pecking occurs independent of FP, is usually

Receive date: 2017-03-08 Accepted date: 2018-06-19

Biographies: Haipeng Shi, $\mathrm{PhD}$ candidate, research interests: animal environmental control and animal welfare, Email: shihaipeng@cau.edu.cn; Weichao Zheng, $\mathrm{PhD}$, Associate Professor, research interests: animal environmental control, agricultural biological environment and energy engineering, Email: weichaozheng@cau.edu.cn; Jiang Tu, Master candidate, research interests: application of electrolyzed water in animal housing.

*Corresponding author: Baoming $\mathbf{L i}, \mathrm{PhD}$, Professor, research interests: animal environmental control, agricultural biological environment and energy engineering. College of Water Resources and Civil Engineering, China Agricultural University, 17 Qinghua East Road, Beijing 100083. Tel: +86-10-62736904, Email: libm@cau.edu.cn. preceded by $\mathrm{FP}$, and is only indirectly associated with $\mathrm{FP}^{[3]}$. Vent pecking is usually termed as cloacal cannibalism, which is characterized by pecking directed specifically at a red area at the top of the cloaca and at small downy feathers below the cloaca and at the underlying tissue surrounding the vent of a conspecific ${ }^{[4]}$. Both severe FP and cloacal cannibalism are generally known as damaging or injurious behavior. Damaging behavior should not be confounded with aggressive pecking, which is for the purpose of establishing and maintaining the dominant hierarchy ${ }^{[5]}$. It is also important to note that not all studies separate VP from FP, so in some cases feather damage includes feather damage to the vent area.

Pathogenesis of both FP and VP amongst hens is still largely unclear and unpredictable, although many studies have been carried out on the possible causative factors, including genetic background, early-life history and environmental factors ${ }^{[3,6]}$. Feather pecking is considered to be a form of redirected pecking ${ }^{[4,7]}$. Two different hypotheses are applied to explain the reasons for directly inducing FP. One believes that FP originates from the peck at the substrate during dustbathing, while the other claims that it derives from foraging behavior ${ }^{[8]}$. Both VP and cloacal cannibalism are associated with FP and share the common risk factors for the development of $\mathrm{FP}^{[9]}$.

In practice, beak trimming has been used as a common technique to prevent or reduce FP and cannibalism ${ }^{[10]}$. But its effectiveness is not consistent, because of different housing systems and varieties of administrative measures, combined stocking density and flock size, adoption of different strains, and 
experimental conditions across different studies ${ }^{[11]}$. Moreover, beak trimming has been criticized because it is contradictory to the conception of animal welfare. Studies have given neurological evidence that the beak trimming procedure itself can cause both acute and chronic pain of hens. Furthermore, adverse impacts on the beak function and sensitivity limit the freedom of expression of normal behaviors ${ }^{[12]}$. A general ban on beak trimming already exists in European Union countries. Dimming the light or altering the light color is frequently used to control FP and cannibalism $^{[10,11,13]}$. The objective is to create visual impairment and diminish the visual detection between each other ${ }^{[11,13]}$. This controlled approach, however, is also questionable. Dimming the light or altering the spectrum may result in abnormal eye development of chickens, and skeletal dysplasia due to the inhibiting activity of laying hens ${ }^{[14]}$. Meanwhile, it is not convenient for the farmers to inspect the flocks. Limited studies have been conducted on the risk factors or diminishing measures of VP of PS layer breeders in colony cages. Published literatures have indicated that the key stimulus for VP is the exposure of the cloacal mucosa membrane of attracted hens during the egg laying period $^{[15]}$. Providing enriched environments for appropriate exploratory stimuli may reduce the likelihood of FP developing. It has been shown that oviposition occurring outside the nest boxes would raise the risk of being pecked at the cloacal or the surrounding areas ${ }^{[16]}$.

New approaches to control injurious pecking in the alternative system of colony cages for natural mating are desired in China, and providing nest boxes may be an effective and welfare-oriented method. The objective of this experiment was to investigate the effects on FP and VP behaviors by providing PS layer breeders with nest boxes in colony cages for natural mating.

\section{Materials and methods}

\subsection{Hens and housing system}

The experiment was conducted in a layer breeder house, with the capacity to accommodate approximately 28000 laying hens in North China. The house is $104 \mathrm{~m} L \times 12 \mathrm{~m} \mathrm{W \times 6} \mathrm{m} \mathrm{H}$, with 8 rows of four-tier stacked colony cages (608 cages in all) along the length of the house. Each cage of $4.80 \mathrm{~m} L \times 1.20 \mathrm{~m} W \times 0.71 \mathrm{~m} H$ confined 10 roosters and 90 laying hens, with a floor area of approximately $576 \mathrm{~cm}^{2}$ per bird. Beak-trimmed Hy-Line browns (Rhode Island Reds X Light Sussex) were used in this study. The experiment was carried out with hens from the age of 37 weeks to 53 weeks.

All the hens in the fourth-tier cages were involved in the experiment. They were divided into two groups from the middle aisle. One group (the treatment) was raised in the colony cages in which two red identical gregarious nest boxes were provided at the age of 37 weeks. Each nest box was $0.90 \mathrm{~m} L \times 0.40 \mathrm{~m} W \times 0.60 \mathrm{~m} \mathrm{H}$ and made of polyethylene resin materials. The two nest boxes were placed at the left end of each colony cage (Figure 1). The other group was raised in the unfurnished colony cages as the control. For each group, six cages (the $1^{\text {st }}, 2^{\text {nd }}, 10^{\text {th }}, 11^{\text {th }}, 18^{\text {th }}$ and $19^{\text {th }}$ cages counting from one end of the house to the other) were selected from each row ( 2 rows for each group) and 12 hens in each of the 6 cages were randomly chosen to be marked with large plastic tags on both wings. These 288 hens (144 for each group) were used as the focal hens for behavioral observation and other tests in this experiment. Throughout the study, the light went on at $4: 30 \mathrm{~h}$ and off at $18: 30 \mathrm{~h}(16 \mathrm{~L}: 8 \mathrm{D})$, with a supplement of two hours of light during the night (starting at 22:30 h). The light intensity was measured at the front of the fourth tier colony cages facing outside at the height of the hens and had an average value of $5.8 \mathrm{~lx}$. The average air temperature at the height of the fourth tier was maintained between $16^{\circ} \mathrm{C}$ and $23^{\circ} \mathrm{C}$. Food and water were supplied ad libitum and automatically, eggs and feces were collected on conveyor belts. All hens were subjected to the same standard management regime throughout the experiment.

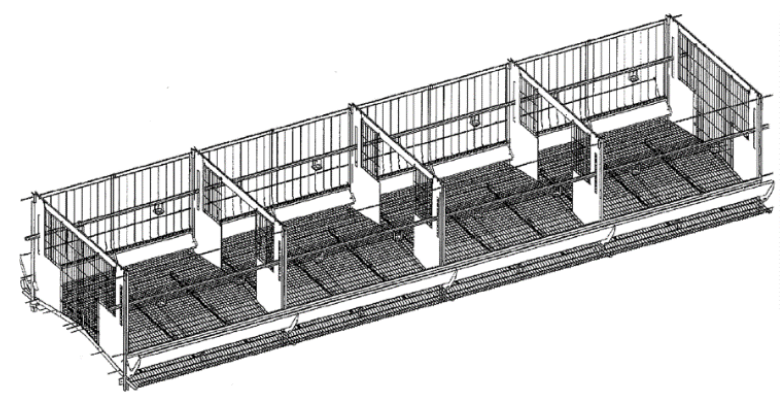

Figure 1 Schematic of the colony cage for natural matting (Hytem Co., Ltd., Gifu, Japan)

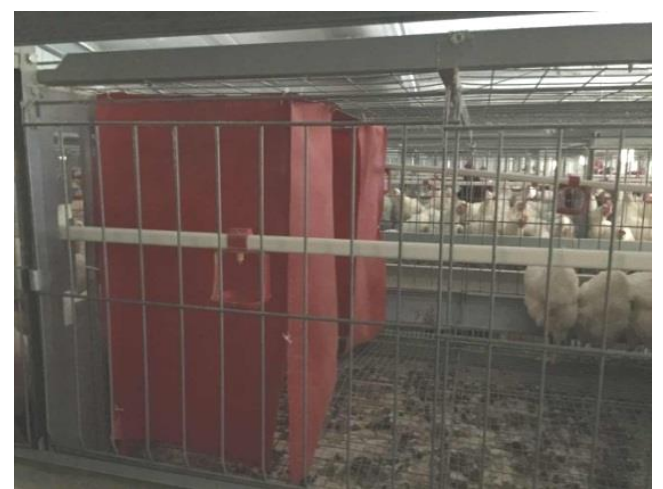

Figure 2 Nest boxes in the colony cage for natural matting

\subsection{Usage of the nest boxes}

A wide-angle infrared camera was set in the top center of each nest for video recording. The hens were not provided nest boxes until the age of 37 weeks old, and the video acquisition was carried out at the age of 41 weeks, 47 weeks and 53 weeks. The main egg-laying period, which was the $5 \mathrm{~h}$ after the lights went on, was filmed on three consecutive days. Scan sampling method was applied to record the number of hens in all nests every $10 \mathrm{~min}$ during each 5-h video acquisition period. The number of nest visits (with entrance and exit) and eggs laid in each nest box were also recorded during the experiment.

\subsection{Behavioral observation}

Behavioral observation was conducted by two trained people when the birds' ages were 41 weeks, 47 weeks and 53 weeks. Observation principles were brought into correspondence with each other before each observation. Each observer carried out one observation cycle for each of the 3 weeks. The total of 288 focal hens were covered in an observation cycle for a duration of two consecutive days. On each day, one person observed three cages from the treatment group in the morning and another three cages from the control group in the afternoon, while the other person carried out the observation in the opposite order. During the observation, the two people sat in an elevated chair in front of the cage for a clear view covering the whole cage. They would keep quiet for $10 \mathrm{~min}$ before starting each observation to minimize any influence caused by their presence. For each cage, the observer recorded the bird behaviors separately from tag number 1 to 12 , lasting $5 \mathrm{~min}$ for each bird. The whole body of the bird was divided into 11 regions: head, upper neck, back, rump, tail, belly 
(abdomen), breast, under neck (front side of the neck), wings-primary feathers, wings-coverts and legs ${ }^{[17]}$. All given and received pecks to the eleven feather areas were counted and classified into gentle and severe pecks based on the observer's judgment of the degree of vigorousness. Aggressive pecks were also recorded.

Table 1 Definitions of the measured behaviors

\begin{tabular}{cl}
\hline \multicolumn{1}{c}{ Behavior } & \multicolumn{1}{c}{ Definition } \\
\hline Gentle feather peck & $\begin{array}{l}\text { Pecks characterized by slow, calm forward thrusts of } \\
\text { the beak, followed by slow, calm withdrawal. Not } \\
\text { resulting in feathers being pulled out and usually } \\
\text { without reaction from the recipient bird. Generally } \\
\text { performed in multiple bouts. } \\
\text { Secks characterized by quick, forceful forward thrusts } \\
\text { Peather peck } \\
\text { of the moderately parted beak, followed by quick, } \\
\text { forceful withdrawal. The feather-pecking bird } \\
\text { advances with the head kept lowered and often the } \\
\text { body is also placed in a lowered position. } \\
\text { Pecks were directed mainly at the head or other parts } \\
\text { of the facial region, given in a downward direction and } \\
\text { were always severe, fast and singular. When the } \\
\text { attacked bird tried to escape, an aggressive peck could } \\
\text { be directed to another part of its body }\end{array}$ \\
\hline
\end{tabular}

\subsection{Mortality and cannibalistic injuries}

During the period of experiment, the numbers of dead hens in both groups were recorded and each dead bird was observed to determine if the death had been caused by cannibalism. The number of cannibalistic injuries in all cages was also recorded. Cannibalistic injuries were defined as bleeding injuries caused by another hen's pecking.

\subsection{Data analysis}

In this experiment, FP frequency was averaged as the number of FPs/5-min per bird. Utilization rates of nest boxes were calculated as the ratio of the number of eggs collected in nest boxes and eggs collected in the whole cage. Mortality rates were defined as the ratio of deaths caused by cloacal cannibalism and all hens in the whole tested cages. Each parameter was tested for normality and heterogeneity of variance prior to analysis. When data were not normally distributed and could not be solved by transformation, non-parametric statistics would be used for analysis. The recordings of FP activity were non-normally distributed even after transformation. Therefore, Man-Whitney U tests were used to identify the effect of treatment on the number of FPs. One-way analysis of variance was used to investigate the effects of the existence of nest boxes on cannibalistic injuries and total mortality rate caused by cloacal cannibalism.

\section{Results and discussion}

\subsection{Usage of nest boxes}

As shown in Table 2, the number of eggs, the number of hens, and the number of nest visits gradually increased after the installation of nest boxes at the age of 37 weeks. The average utilization rates of nest boxes were $36.75 \%, 44.75 \%$ and $50.38 \%$ at the ages of 41 weeks, 47 weeks and 53 weeks, respectively.

Genetically, hens usually prefer to pursue discrete enclosed spaces in which to lay their eggs ${ }^{[18]}$. The installation of the nest boxes in the cages was expected to increase the possibility for satisfying this natural need of nesting behavior. The hens' motivation to access a nest box increases when the time for egg laying approaches. They will spare no effort to squeeze through narrow gaps ${ }^{[19]}$ and even to open a door to gain access to a pen containing an enclosed nest box ${ }^{[20]}$. Some behaviors showing signs of frustration would happen in the absence of an appropriate nesting environment, such as pacing, 'gakel' vocalizations, a prolonged searching phase and a shorter sitting time prior to oviposition $^{[21]}$. It has been reported that the incidence of floor eggs in cages with nest boxes ranged from $10 \%-57 \%^{[22]}$. In this study, more than half of the eggs $(50.38 \%)$ were laid in the nest boxes after 16 weeks of their installation, but the number of eggs laid outside of the nest boxes was still considerable. Two reasons may explain why nearly half of the eggs were laid outside of the nest boxes in this experiment. Firstly, a hen might lack an individual nest preference and attracted by the presence of nest-like features elsewhere in the cage ${ }^{[23]}$. Secondly, since the nest box was installed at the age of 37 weeks, the hens may have already formed a habit of laying eggs in some particular repeated locations without a nest for consistent egg-laying sites ${ }^{[24]}$. Thus, in any further study, the nest boxes should be installed before the hens commence laying their eggs.

Table 2 Use of nest boxes for hens at the ages of 41 weeks, 47 weeks and 53 weeks

\begin{tabular}{lcccccccc}
\hline \multirow{2}{*}{ Use of Nest Boxes } & \multicolumn{2}{c}{41 weeks } & & \multicolumn{2}{c}{47 weeks } & & \multicolumn{2}{c}{53 weeks } \\
\cline { 2 - 3 } \cline { 7 - 8 } \cline { 7 - 8 } & Mean & SEM & & Mean & SEM & & Mean & SEM \\
\hline Number of eggs & 29.4 & 2.8 & & 35.8 & 3.1 & & 40.3 & 3.6 \\
Number of hens & 21.0 & 1.2 & & 29.0 & 1.2 & & 33.0 & 1.4 \\
Number of nest visits & 201.7 & 11.3 & & 280.0 & 15.6 & 341.1 & 17.2 \\
\hline
\end{tabular}

Note: The mean number and SEM value of eggs, hens and nest visits per day per cage are presented.

\subsection{Pecking behavior}

The FP frequency of hens for both the treatment and the control groups at the ages of 41 weeks, 47 weeks and 53 weeks are given in Figure 3. More FP activities happened in the control group compared to the treatment group. When combining severe and gentle pecks to ensure a sufficient number of pecks, it was found that the total number of gentle and severe received FPs was larger than the total number of gentle and severe FPs given in this experiment. A uniform difference in given and received pecks can be summarized in the frequency of aggressive pecks.

As shown in Table 3, for both gentle given and received FPs, hens in the control group were involved significantly more often than hens in the treatment group at the ages of 41 weeks, 47 weeks, and 53 weeks $(p<0.05)$. Both given and received gentle FPs showed a decreasing trend with increasing age for both groups. No significant difference in the numbers of severe given or received FPs was found at the age of 41 weeks, but much less severe FPs in the treatment group happened than the control group at the age of 47 weeks and 53 weeks $(p<0.05)$. It was further noticed that there was no significant difference in aggressive pecks given or received at the age of 47 weeks, nor significant difference in aggressive pecks given at the age of 53 weeks. But hens in the treatment group received significantly fewer aggressive pecks than hens in the control group at the ages of 41 weeks and 53 weeks $(p<0.05)$ and gave significantly fewer aggressive pecks at the age of 41 weeks $(p<0.05)$.

The results of the experiment confirmed our hypothesis that hens without access to nest boxes in a colony cage performed more active FP activities. For laying hens in cages, environmental enrichment has been widely employed to alleviate FP behaviors. Giving stimulation to those hens unable to fulfill inherent drives due to environmental limitations, such as providing foraging materials ${ }^{[25]}$ and offering access to perches from an early age ${ }^{[16]}$, can reduce abnormal FP frequency. Other manipulable stimuli like novel objects, food, pictures, sounds, string, chains, or beads 
can as well be regarded as putative enrichment ${ }^{[26]}$. Chow and $\operatorname{Hogan}^{[27]}$ suggested that hens would exhibit less conspecific pecking when being given the opportunity to express in exploratory pecking in an environment with an exploratory-rich device. Gunnarsson et al. ${ }^{[16]}$ have reported that providing hens access to perches from an early age (no later than four weeks) mitigated the occurrence of floor eggs during the early laying period, and FP and cloacal cannibalism during the entire laying period. The installation of nest boxes in colony cages for natural mating in our
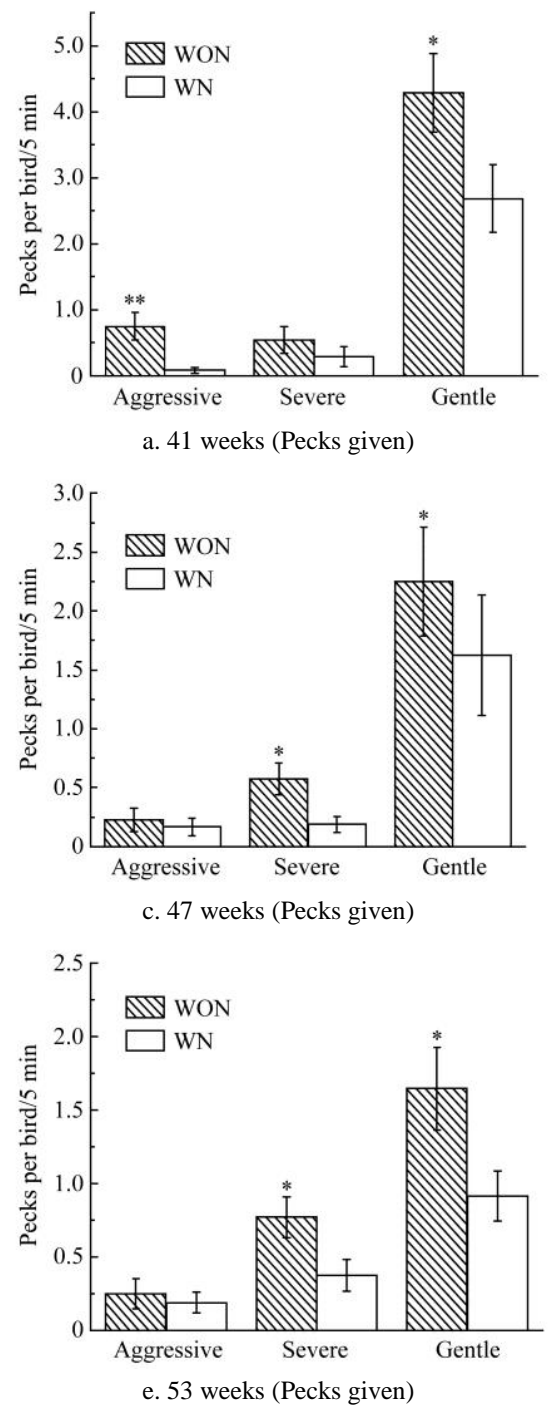

Note: $*: p<0.05, * * p<0.01$.

Figure 3 Mean number of aggressive, severe and gentle pecks per bird per cage during a 5-min observation at the ages of 41 weeks, 47 weeks and 53 weeks in the cages without nest boxes (WON) and with nest boxes (WN)

Table 3 Significant difference analysis results of FPs between treatment and control groups (at the $5 \%$ significance level)

\begin{tabular}{ccccc}
\hline \multirow{2}{*}{ Gentle } & Given/received FPs & 41 weeks & 47 weeks & 53 weeks \\
& Given & Yes & Yes & Yes \\
& Received & Yes & Yes & Yes \\
\hline \multirow{2}{*}{ Severe } & Given & No & Yes & Yes \\
& Received & No & Yes & Yes \\
\hline \multirow{2}{*}{ Aggressive } & Given & Yes & No & No \\
& Received & Yes & No & Yes
\end{tabular}

Note: Yes means the FPs that happened in the treatment were significantly less than the control; No means of significant difference was found between the treatment and the control. study, which can be regarded as one type of environmental enrichment device, can not only satisfy the need of natural nesting behavior expression of hens, but also provide them opportunities for exploratory behaviors. Comparatively, hens in the control group were reared in a barren and invariant environment, which minimized the opportunities for exploration. Such environmental impoverishment could compromise the hens' welfare by increasing fearfulness, depression, FP, and cognitive impairment as well as leading to reduced productivity ${ }^{[28]}$.
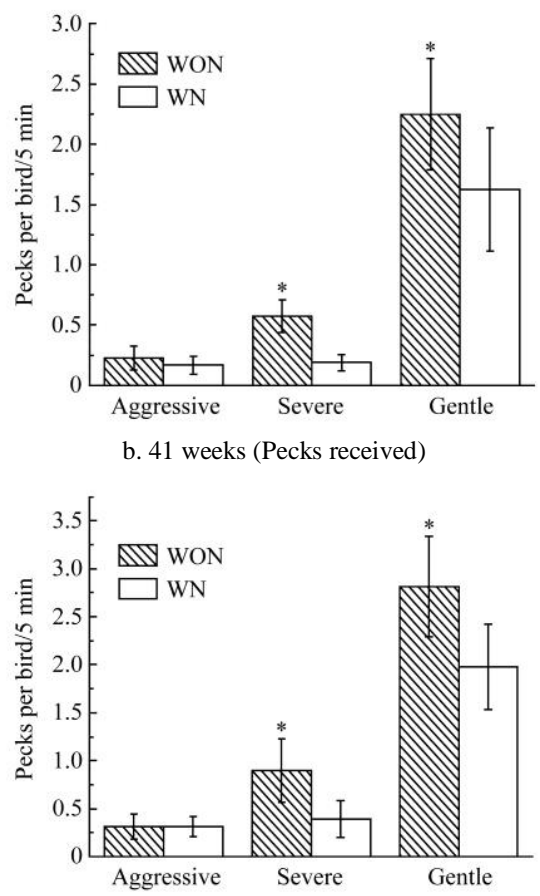

d. 47 weeks (Pecks received)

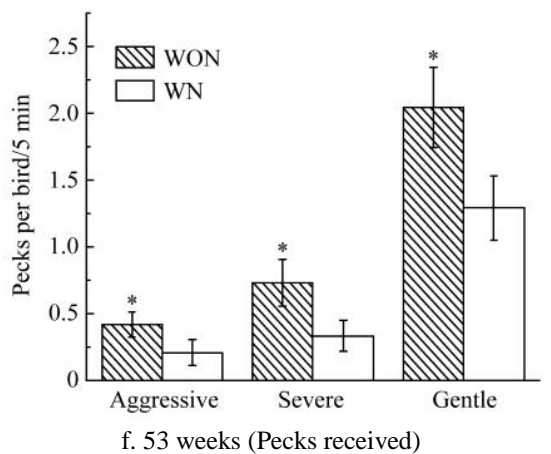
the alternative housing system for PS layer breeders. Several possible reasons can explain why the existence of nest boxes in colony cages has affected FP control in this study. Firstly, hens have good color vision, and color is considered likely to be an important attribute of any enrichment device. Roper suggested that red-colored water or food or objects were particularly attractive and chosen preferentially by hens ${ }^{[29]}$. The conspicuous red color of the nest boxes would be intuitively more attractive and sustained appreciable interest of the hens. During behavioral observation, it was noticed that a large proportion of hens were found to express a curious state that they raised their heads and stared at the nest boxes and most of them preferred to peck at the nest boxes throughout the entire experiment. The hens 
consistently spent a longer time near the nest boxes, therefore, the attention and conspecific pecking transferred to pecks toward nest boxes. Secondly, the installation of nest boxes in colony cages provided hens opportunities to perform their natural nesting behavior. In this study, more than half of the hens tested in the experiment laid eggs in nest boxes. Several studies have shown that most eggs are laid during the first $5 \mathrm{~h}$ after the light is switched ${ }{ }^{[30]}$. The time hens spend in nest boxes for egg laying have been measured. Wall et al. ${ }^{[31]}$ reported that hens occupied nest boxes for about $40 \mathrm{~min} / \mathrm{d}$, while Cronin et al. ${ }^{[22]}$ reported hens were in nest boxes for about $29 \mathrm{~min}$ before egg laying and remained there for a further $6.5 \mathrm{~min}$ after oviposition before leaving. Based on our observation, there were always some hens exploring and staying in the nest boxes from light on to light off. A hen in a nest box was concealed from her flock-mates (temporarily, at least), which would alleviate the competition for food or water. This could also account for the effect of nest boxes on FP behavior control.

\subsection{Mortality and cannibalistic injuries}

The total number of deaths and cannibalistic injuries caused by VP per cage of the two different groups is shown in Figure 4. Dying from hemorrhage caused by VP was classified as a case of cloacal cannibalism. During the experiment, the first death from cloacal cannibalism occurred in the control group when the hens were 39 weeks old. Cloacal cannibalism and cannibalistic injuries occurred in both the treatment group and the control group. However, the total mortality rate caused by cloacal cannibalism from 38 weeks to 55 weeks was significantly higher in the control group $(13.3 \%)$ compared to the corresponding mortality rate in the treatment group $(5.6 \%)(p<0.01)$. There were significantly more injuries inflicted by vent pecking recorded in groups reared in the control group $(p<0.01)$.

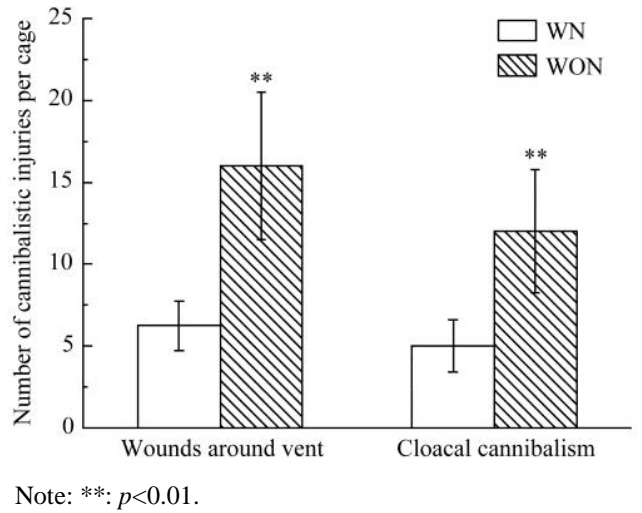

Figure 4 Total number of cloacal cannibalism cases and wounds around vent per cage of two different groups of hens in cages without nest boxes (WON) and with nest boxes (WN) during the period of the experiment

This study showed that hens having no access to nest boxes had significantly higher mortality rates caused by VP and more cannibalistic injuries. Whereas VP is directed toward hens, no studies found that males are victims of cloacal cannibalism. Cannibalism toward the cloaca of females may originate from the attraction to the cloaca when it changes its appearance at the onset of laying eggs. It has been suggested that the key stimulus for VP is the exposure of cloacal mucosa membrane of attracted hens during the egg laying period ${ }^{[9,15]}$. To demonstrate this idea, several studies have shown that the cloaca was everted for an average of $22 \mathrm{~s}$ and $44 \mathrm{~s}$ following egg expulsion, in two genetic strains of White Leghorn, respectively, while VP was prevalent in the latter $\operatorname{strain}^{[32]}$. Although there may exist other strain differences, it seems that cloacal prolapse following oviposition can be a risk factor for cloacal cannibalism. With oviposition occurring outside the nest boxes, the layer would have a higher risk of being pecked at the cloacal or the surrounding areas ${ }^{[16]}$. In this study, as was expected, providing nest boxes and promoting their use by hens was beneficial for reducing the visibility of the cloaca during oviposition, thereby reducing the risk of VP. Gregarious nests in this study were acceptable and well used by hens. Nests provided in this study were enclosed and relatively dark rather than open or provided with additional lighting. An epidemiological study has shown that leaving an area unlit to encourage the use of nest boxes resulted in less VP than dimly lit nests ${ }^{[1]}$. Factors that increase nest utilization, including rearing with perches from an early age ${ }^{[33]}$, will also reduce oviposition in exposed locations, thereby reducing the risk of VP. Encouraging more use of nest boxes by placing nests at the ends of cages rather than in the center may also be favorable in reducing the probability that hens' oviposition will be witnessed by other hens or come into contact with their cloacae even shortly after oviposition ${ }^{[34]}$. So, it is feasible to provide hens with nest boxes designed to minimize visibility of the cloaca mucosa during egg laying.

\section{Conclusions}

Due to increasing labor cost, an unfurnished colony cage system for natural mating of PS layer breeders has been widely applied in China. In this study, nest boxes were provided in the alternative system to investigate their effects on mitigating FP and VP behaviors of hens. Providing nest boxes not only allows expression of natural nesting behavior but also reduces FP behavior and alleviates hens' injury. It can provide a supernumerary profit of constructing micro-environments that may be perceived as safer, thus stimulating the performance of comfort behaviors, and ultimately increase welfare. Providing nest boxes is an effective method to eliminate the problems caused by FP and VP behaviors, and to reduce the mortality and cannibalistic injuries. However, providing nest boxes cannot solve the FP and VP problem in the system. Additional management strategies for reducing the damage caused by FP and VP in this system should be explored in further studies.

\section{Acknowledgements}

This research was funded by China Agricultural Research System (CARS-40) and Beijing Science \& Technology Committee Special Project (Z171100002217018). The authors would also like to thank the manager, Zhi Wang, and the other staff of Hebei Huayu Poultry Breeding Co. Ltd., Handan, Hebei, China. Thanks also go to the students in the department who helped to take care of the hens.

\section{[References]}

[1] Pötzsch C J, Lewis K, Nicol C J, Green LE. A cross-sectional study of the prevalence of vent pecking in laying hens in alternative systems and its associations with feather pecking, management and disease. Applied Animal Behaviour Science, 2001; 74(4): 259-272.

[2] Rodenburg T B, Koene P. Comparison of individual and social feather pecking tests in two lines of laying hens at ten different ages. Applied Animal Behaviour Science, 2003; 81(2): 133-148.

[3] Allen J, Perry G C. Feather pecking and cannibalism in a caged layer flock. British Poultry Science, 1975; 16(5): 441-451.

[4] Savory C J. Feather pecking and cannibalism. Worlds Poultry Science Journal, 1995; 51(2): 215-219. 
[5] Duncan I J H, Wood-Gush D G M. Frustration and aggression in the domestic fowl. Animal Behaviour, 1971; 19(3): 500-504

[6] Rodenburg T B, Komen H, Ellen E D, Uitdehaag K A, Arendonk J A. Selection method and early-life history affect behavioural development, feather pecking and cannibalism in laying hens: A review. Applied Animal Behaviour Science, 2008; 110(3): 217-228.

[7] Rodenburg T B, Koene P. The impact of group size on damaging behaviours, aggression, fear and stress in farm animals. Applied Animal Behaviour Science, 2007; 103(3-4): 205-214.

[8] Blokhuis H J, Arkes J G. Some observations on the development of feather-pecking in poultry. Applied Animal Behaviour Science, 1984; 12(1-2): 145-157.

[9] Lambton S L, Knowles T G, Yorke C, Nicol C J. The risk factors affecting the development of vent pecking and cannibalism in free-range and organic laying hens. Animal Welfare, 2015; 24(1): 101-111.

[10] Lambton S L, Knowles T G, Yorke C, Nicol C J. The risk factors affecting the development of gentle and severe feather pecking in loose housed laying hens. Applied Animal Behaviour Science, 2010; 123(1-2): $32-42$.

[11] Nicol C J, Bestman M, Gilani A M, Haas E N D, Jong I C D, Lambton S, et al. The prevention and control of feather pecking: application to commercial systems. World's Poultry Science Journal, 2013; 69(4): 775-788.

[12] Freire R, Eastwood M A, Joyce M. Minor beak trimming in chickens leads to loss of mechanoreception and magnetoreception. Journal of Animal Science, 2011; 89(4): 1201-1206.

[13] Mohammed H H, Grashorn M A, Bessei W. The effects of lighting conditions on the behaviour of laying hens. Archiv Fur Geflugelkunde, 2010; 74(3): 197-202.

[14] Prescott N B, Wathes C M, Jarvis J R. Light, vision and the welfare of poultry. Animal Welfare, 2003; 12(2): 269-288.

[15] Yngvesson J, Keeling L J, Newberry R C. Individual production differences do not explain cannibalistic behaviour in laying hens. British Poultry Science, 2004; 45(4): 453-462.

[16] Gunnarsson S, Keeling L J, Svedberg J. Effect of rearing factors on the prevalence of floor eggs, cloacal cannibalism and feather pecking in commercial flocks of loose housed laying hens. British Poultry Science, 1999; 40(1): 12-8.

[17] Bilcík B, Keeling L J. Changes in feather condition in relation to feather pecking and aggressive behaviour in laying hens. British Poultry Science, 1999; 40(4): 444-451.

[18] Weeks C A, Nicol C J. Behavioural needs, priorities and preferences of laying hens. Worlds Poultry Science Journal, 2006; 62(2): 296-307.

[19] Cooper J J, Appleby M C. Demand for nest boxes in laying hens.
Behavioural Processes, 1996; 36(2): 171-182.

[20] Cooper J J, Appleby M C. The value of environmental resources to domestic hens: a comparison of the work-rate for food and for nests as a function of time. Animal Welfare, 2003; 12(1): 39-52.

[21] Cooper J J, Albentosa M J. Behavioural priorities of laying hens Avian \& Poultry Biology Reviews, 2003; 14(3): 127-149.

[22] Cronin G M, Butler K L, Desnoyers M A, Barnett J L, Witkowski A The use of nest boxes by hens in cages: what does it mean for welfare? Animal Science Papers \& Reports, 2005; 23(1): 121-128.

[23] Wall H. Production performance and proportion of nest eggs in layer hybrids housed in different designs of furnished cages. Poultry Science, 2011; 90(10): 2153-2161.

[24] Rietveld P B, Blokhuisb H J, Wiepkemaa P R. Egg-laying behaviour and nest-site selection of domestic hens kept in small floor-pens. Applied Animal Behaviour Science, 1985; 14(1): 75-88.

[25] Haas E N D, Bolhuis J E, Kemp B, Groothuis T G G, Rodenburg T B Parents and early life environment affect behavioral development of laying hen chickens. PLoSOne, 2014; 9(3): e90577.

[26] Gao W, Jjr F, Robinson F E, Cook H. Effect of stocking density on the incidence of usage of enrichment devices by white Leghorn hens. The Journal of Applied Poultry Research, 1994; 3(4): 336-341.

[27] Chow A, Hogan J A. The development of feather pecking in Burmese red jungle fowl: The influence of early experience with exploratory-rich environments. Applied Animal Behaviour Science, 2005; 93(3): 283-294.

[28] Appleby M C, Hughes B O, Elson H A. Poultry production systems: Behaviour, management and welfare. Livestock Production Science, 1993; 36(2): 196-197.

[29] Roper T J, Marples N M. Colour preferences of domestic chicks in relation to food and water presentation. Applied Animal Behaviour Science, 1997; 54(2): 207-213.

[30] RiberA B. Development with age of nest box use and gregarious nesting in laying hens. Applied Animal Behaviour Science, 2010; 123(1): 24-31.

[31] Wall H, Tauson R, Elwinger K E. Effect of nest design, passages, and hybrid on use of nest and production performance of layers in furnished cages. Poultry Science, 2002; 81(3): 333-339.

[32] Hori H, Kamei I. Research on time of contraction of the cloaca after oviposition. Japanese Poultry Science, 1986; 23: 379-380.

[33] Appleby M C, Duncan I J H, McRae H E. Perching and floor laying by domestic hens: Experimental results and their commercial application. British Poultry Science, 1988; 29(2): 351-357.

[34] Huber-Eicher B. The effect of early colour preference and of a colour exposing procedure on the choice of nest colours in laying hens. Applied Animal Behaviour Science, 2004; 86(1-2): 63-76. 